\title{
Poisson reduction and the Hamiltonian structure of the Euler-Yang-Mills equations
}

\author{
François Gay-Balmaz and Tudor S. Ratiu
}

\begin{abstract}
The problem treated here is to find the Hamiltonian structure for an ideal gauge-charged fluid. Using a Kaluza-Klein point of view, we obtain the non-canonical Poisson bracket and the motion equations by a Poisson reduction involving the automorphism group of a principal bundle.
\end{abstract}

\section{Introduction}

The motion equations of an ideal incompressible fluid in a Riemannian manifold $(M, g)$ are given by the Euler equations

$$
\frac{\partial v}{\partial t}+\nabla_{v} v=-\operatorname{grad} p
$$

where the Eulerian velocity $v$ is a divergence free vector field, $p$ is the pressure and $\nabla$ is the Levi-Civita covariant derivative associated to $g$. From $[\mathbf{A}]$ it is known that equations (1.1) are formally the spatial representation of the geodesic motion on the volume-preserving diffeomorphism group $\mathcal{D}_{\mu}(M)$ of $M$ with respect to the $L^{2}$ Riemannian metric. See also $[\mathbf{A M}], \S 5.5 .8$, for a quick exposition of this fact and $[\mathbf{E M}]$ for the analytic formulation and many rigorous results concerning the Euler and Navier-Stokes equations derived from this geometric point of view. From the Hamiltonian perspective, equations (1.1) are the Lie-Poisson equations on the Lie algebra $\mathfrak{X}_{\text {div }}(M)$ of $\mathcal{D}_{\mu}(M)$, consisting of divergence free vector fields. Here, $\mathfrak{X}_{\text {div }}(M)$ is identified with its dual by the weak $L^{2}$-pairing.

1991 Mathematics Subject Classification. Primary 37K65, 53C80, 70S15; Secondary 53D17, 76W05.

Key words and phrases. Lie-Poisson equations, Euler-Yang-Mills equations, automorphism group, gauge group, reduction, Kaluza-Klein metric, Poisson bracket.

The authors were supported in part by the Swiss National Science Foundation Grant \#200021-109111.

(c)0000 (copyright holder) 
In [MRW, MWRSS], this approach is generalized to the case of the motion of an ideal compressible adiabatic fluid

$$
\left\{\begin{array}{l}
\frac{\partial v}{\partial t}+\nabla_{v} v=\frac{1}{\rho} \operatorname{grad} p \\
\frac{\partial \rho}{\partial t}+\operatorname{div}(\rho v)=0 \\
\frac{\partial \sigma}{\partial t}+\mathbf{d} \sigma(v)=0
\end{array}\right.
$$

where $\rho$ is the mass density, $\sigma$ is the specific entropy and $p$ is the pressure. In this case, the configuration space is the full diffeomorphism group $\mathcal{D}(M)$ and equations (1.2) are obtained via Lie-Poisson reduction for semidirect products.

In this paper we generalize the previous approach to the case of a classical charged ideal fluid. More precisely, using a Kaluza-Klein point of view, we obtain the motion equations by Poisson reduction. Therefore we need to consider on $M$ a $G$-principal bundle $P \rightarrow M$ and to enlarge the configuration space from the group of diffeomorphisms to the product of the group of automorphisms of $P$ with the field variables. This point of view is directly inspired by the paper $[\mathbf{M}]$ on the motion of a particle in a Yang-Mills field.

If $G=S^{1}$ we get the Euler-Maxwell equations describing the motion of an electrically charged fluid. The Euler-Maxwell equations are given by the system

$$
\left\{\begin{array}{l}
\frac{\partial v}{\partial t}+\nabla_{v} v=\frac{q}{m}(\mathbf{E}+v \times \mathbf{B})-\frac{1}{\rho} \operatorname{grad} p \\
\frac{\partial \rho}{\partial t}+\operatorname{div}(\rho v)=0 \\
\frac{\partial \sigma}{\partial t}+\mathbf{d} \sigma(v)=0, \\
\frac{\partial \mathbf{E}}{\partial t}=\operatorname{curl} \mathbf{B}-\frac{q}{m} \rho v, \quad \frac{\partial \mathbf{B}}{\partial t}=-\operatorname{curl} \mathbf{E}, \\
\operatorname{div} \mathbf{E}=\frac{q}{m} \rho, \quad \operatorname{div} \mathbf{B}=0,
\end{array}\right.
$$

where $v$ is the Eulerian velocity, $\mathbf{E}$ is the electric field, $\mathbf{B}$ is the magnetic field, and the constant $q$ is the charge per unit mass. The Hamiltonian structure of the Euler-Maxwell equation was derived in the paper [MWRSS] and is closely related to the Hamiltonian structure of the Maxwell-Vlasov equations (see $[\mathbf{M W}]$ ).

In the general case of a Lie group $G$, we will show that the Poisson reduction leads to the equation for an ideal compressible adiabatic fluid carrying a gaugecharge, as given in $[\mathbf{G H K}]$. We call these equations the Euler-Yang-Mills equations. The standard examples are obtained for $G=\mathrm{SU}(2)$ or $G=\mathrm{SU}(3)$.

The physical interpretation of the point of view given in this paper is the following. The evolution of the fluid particles as well as of the gauge-charge density of the fluid is given by a curve $\psi_{t}$ in the automorphism group. In fact $\psi_{t}$ is the flow of a time-dependent vector field $U_{t}$ on the principal bundle $P$. This vector field induces a time-dependent vector field $v_{t}$ on $M$, which represents the Eulerian velocity of the fluid. Given the evolution of the magnetic potential $\mathcal{A}_{t}$ and of the mass density $\rho_{t}$, the vector field $U_{t}$ induces also a Lie algebra valued and time-dependent function $\rho_{t} \mathcal{A}_{t}\left(U_{t}\right)$, which represents the gauge-charge density of 
the fluid. Note the analogy with the Kaluza-Klein point of view for the charged particle in a Yang-Mills field (see $[\mathbf{M}]$ ).

The plan of the paper is as follows. Section 2 recalls some needed facts about principal bundles, connections, automorphisms, and gauge groups. Section 3 recalls the Hamiltonian formulation of the Maxwell equations and generalizes this to the case of the Yang-Mills fields equations. Section 4 shows that the compressible and incompressible Euler-Yang-Mills equations are Lie-Poisson equations for a semidirect product associated to the automorphism group of a principal bundle. One of the equations is obtained by conservation of the momentum map associated to the invariance under gauge transformations. We naturally obtain the associated noncanonical Poisson bracket. By applying the general process of reduction by stages, we get some already known results about the Euler-Maxwell equations. We also show that the two different Poisson brackets derived in [GHK] and in [MWRSS] are in fact obtained by Poisson reduction, at different stages, of the same canonical Poisson structure.

\section{Connections, automorphisms and gauge transformations}

In this section we fix our conventions on principal bundles and connections. Consider a smooth free and proper right action

$$
\Phi: G \times P \rightarrow P, \quad(g, p) \mapsto \Phi_{g}(p)
$$

of a Lie group $G$ on a manifold $P$. Thus we get the principal bundle

$$
\pi: P \rightarrow M:=P / G,
$$

where $M$ is endowed with the unique manifold structure for which $\pi$ is a submersion.

Recall that the adjoint vector bundle is

$$
\text { Ad } P:=P \times_{G} \mathfrak{g} \rightarrow M,
$$

where the quotient is taken relative to the right action $(g,(p, \xi)) \mapsto\left(\Phi_{g}(p), \operatorname{Ad}_{g^{-1}}(\xi)\right)$. The elements of $\operatorname{Ad} P$ are denoted by $[p, \xi]_{G}$, for $(p, \xi) \in P \times \mathfrak{g}$.

Consider the space $\Omega^{k}(P, \mathfrak{g})$ of $\mathfrak{g}$-valued $k$-forms on $P$ and let $\overline{\Omega^{k}}(P, \mathfrak{g})$ be the subspace of $\Omega^{k}(P, \mathfrak{g})$ consisting of $\mathfrak{g}$-valued $k$-forms $\omega$ such that

(1) $\Phi_{g}^{*} \omega=\operatorname{Ad}_{g^{-1}} \circ \omega$,

(2) if one of $u_{1}, \ldots, u_{k} \in T_{p} P$ is vertical then $\omega\left(u_{1}, \ldots, u_{k}\right)=0$.

$\overline{\Omega^{k}}(P, \mathfrak{g})$ is naturally isomorphic to $\Omega^{k}(M, \operatorname{Ad} P)$, the space of Ad $P$-valued $k$-forms on $M$. Indeed, to each $\omega \in \overline{\Omega^{k}}(P, \mathfrak{g})$ corresponds a $k$-form $\widetilde{\omega} \in \Omega^{k}(M, \operatorname{Ad} P)$ given on $v_{1}, . ., v_{k} \in T_{x} M$ by

$$
\widetilde{\omega}(x)\left(v_{1}, \ldots, v_{k}\right):=\left[p, \omega(p)\left(u_{1}, \ldots, u_{k}\right)\right]_{G},
$$

where $p \in P$ is such that $\pi(p)=x$ and $u_{i} \in T_{p} P$ are such that $T_{p} \pi\left(u_{i}\right)=v_{i}$. We shall use the notation $\overline{\Omega^{0}}(P, \mathfrak{g})=\mathcal{F}_{G}(P, \mathfrak{g})$ and $\Omega^{0}(M, \operatorname{Ad} P)=\Gamma(\operatorname{Ad} P)$.

A principal connection on $P$ is a $\mathfrak{g}$-valued 1-form $\mathcal{A} \in \Omega^{1}(P, \mathfrak{g})$ such that

$$
\Phi_{g}^{*} \mathcal{A}=\operatorname{Ad}_{g^{-1}} \circ \mathcal{A} \text { and } \mathcal{A}\left(\xi_{P}\right)=\xi,
$$

where $\xi_{P}$ is the infinitesimal vector field associated to $\xi$. The set of all connections will be denoted by $\operatorname{Conn}(P)$. It is an affine space with underlying vector space $\overline{\Omega^{1}}(P, \mathfrak{g})$. Recall that a connection induces a splitting $T_{p} P=V_{p} P \oplus H_{p} P$ of the tangent space into the vertical and horizontal subspace defined by

$$
H_{p} P:=\operatorname{ker}(\mathcal{A}(p)) \text {. }
$$


The covariant exterior differential associated to $\mathcal{A}$ is the map $\mathbf{d}^{\mathcal{A}}: \Omega^{k}(P, \mathfrak{g}) \rightarrow$ $\Omega^{k+1}(P, \mathfrak{g})$ defined by

$$
\mathbf{d}^{\mathcal{A}} \omega(p)\left(u_{1}, \ldots, u_{k}\right):=\mathbf{d} \omega(p)\left(\operatorname{hor}_{p}\left(u_{1}\right), \ldots, \operatorname{hor}_{p}\left(u_{k}\right)\right),
$$

where $\operatorname{hor}_{p}\left(u_{i}\right)$ is the horizontal part of $u_{i} \in T_{p} P, i=1, \ldots, k$. Note that for $\omega \in \overline{\Omega^{k}}(P, \mathfrak{g})$ we have $\mathbf{d}^{\mathcal{A}} \omega \in \overline{\Omega^{k+1}}(P, \mathfrak{g})$.

The curvature of the connection $\mathcal{A}$ is, by definition, the 2 -form

$$
\mathcal{B}:=\mathbf{d}^{\mathcal{A}} \mathcal{A} \in \overline{\Omega^{2}}(P, \mathfrak{g}) .
$$

Recall that a principal connection $\mathcal{A}$ on $P$ induces an affine connection and a covariant derivative, denoted respectively by $\nabla^{\mathcal{A}}$ and $\frac{D^{\mathcal{A}}}{d t}$, on the vector bundles Ad $P \rightarrow M$ and $\operatorname{Ad} P^{*} \rightarrow M$ (see $[\mathbf{K N}]$ ).

Given a Riemannian metric $g$ on $M$ and a connection $\mathcal{A}$ on $P$, we can define the covariant codifferential

$$
\delta^{\mathcal{A}}: \overline{\Omega^{k}}(P, \mathfrak{g}) \rightarrow \overline{\Omega^{k-1}}(P, \mathfrak{g}),
$$

see definition 4.2 .8 in $[\mathbf{B}]$.

Given a Riemannian metric $g$ on $M$ and an Ad-invariant inner product $\gamma$ on $\mathfrak{g}$ we can define a Riemannian metric $g \gamma$ on the vector bundles $\Lambda^{k}(M, \operatorname{Ad} P) \rightarrow M$.

Let $M$ be a compact oriented boundaryless manifold. For $\alpha \in \Omega^{k}(M, \operatorname{Ad} P)$ and $\beta \in \Omega^{k+1}(M, \operatorname{Ad} P)$ we have (see Theorem 4.2.9 in [B]) :

$$
\int_{M}(g \gamma)\left(\mathbf{d}^{\mathcal{A}} \alpha, \beta\right) \mu=\int_{M}(g \gamma)\left(\alpha, \delta^{\mathcal{A}} \beta\right) \mu
$$

where $\mu$ denotes the volume form associated to the Riemannian metric $g$.

Given a connection $\mathcal{A}$, a Riemannian metric $g$ on $M$ and an Ad-invariant inner product $\gamma$ on $\mathfrak{g}$, we can define the Kaluza-Klein metric $K_{\mathcal{A}}$ on $T P$ by

$$
K_{\mathcal{A}}\left(u_{p}, v_{p}\right):=g\left(T_{p} \pi\left(u_{p}\right), T_{p} \pi\left(v_{p}\right)\right)+\gamma\left(\mathcal{A}\left(u_{p}\right), \mathcal{A}\left(v_{p}\right)\right) .
$$

We say that a diffeomorphism $\varphi$ of $P$ is an automorphism if $\Phi_{g} \circ \varphi=\varphi \circ \Phi_{g}$, for all $g \in G$. The Fréchet Lie group of all automorphisms is denoted by $\mathcal{A} u t(P)$. An automorphism $\varphi$ of $P$ induces a unique diffeomorphism $\bar{\varphi}$ of $M$ defined by the condition $\pi \circ \varphi=\bar{\varphi} \circ \pi$. The Lie algebra aut $(P)$ consists of $G$-invariant vector fields on $P$. Its (left) Lie bracket is denoted by $[U, V]_{L}$ and is the negative of the usual Jacobi-Lie bracket $[U, V]_{J L}$. For $U \in \mathfrak{a} \mathfrak{t}(P)$ we denote by $[U] \in \mathfrak{X}(M)$ the unique vector field on $M$ defined by the condition

$$
T \pi \circ U=[U] \circ \pi .
$$

The normal subgroup $\mathcal{G} a u(P)$ of gauge transformations contains, by definition, the automorphisms $\varphi$ with $\bar{\varphi}=i d$.

The Lie algebra $\mathfrak{g a u}(P)$ consists of $G$-invariant vertical vector fields on $P$. Therefore when $U \in \mathfrak{g a \mathfrak { u }}(P)$ we have $[U]=0$. Note the identifications

$$
\mathfrak{g a u}(P) \cong \mathcal{F}_{G}(P, \mathfrak{g}) \cong \Gamma(\operatorname{Ad} P) .
$$

Indeed, to $f \in \mathcal{F}_{G}(P, \mathfrak{g})$ we can associate the $G$-invariant vertical vector field $\sigma(f)$ given by

$$
\sigma(f)(p):=f(p)_{P}(p)
$$


The second equivalence is given by the map (2.1). A direct computation shows that $\sigma$ is a Lie algebra isomorphism, that is,

$$
\sigma([f, g])=[\sigma(f), \sigma(g)]_{L} .
$$

If the principal bundle $P \rightarrow M$ is trivial the automorphism group is a semidirect product of two groups :

$$
\mathcal{A} u t(P) \simeq \mathcal{D}(M) \subseteq \mathcal{G} a u(P) .
$$

Duality. Throughout this paper we will identify the cotangent space $T_{\varphi}^{*} \mathcal{A} u t(P)$ with the space of $G$-invariant 1-forms on $P$ along $\varphi \in \mathcal{A} u t(P)$. The duality is given by

$$
\left\langle\mathbf{M}_{\varphi}, U_{\varphi}\right\rangle:=\int_{M} \mathbf{M}_{\varphi}\left(U_{\varphi}\right) \mu,
$$

where $\mathbf{M}_{\varphi} \in T_{\varphi}^{*} \mathcal{A} u t(P)$ and $U_{\varphi} \in T_{\varphi} \mathcal{A} u t(P)$. Note that in this formula we used the fact that $\mathbf{M}_{\varphi}\left(U_{\varphi}\right)$ is a smooth function on $P$ that does not depend on the fiber variables and hence induces a unique smooth function on $M$. In particular we have $\mathfrak{a u t}(P)^{*}=\Omega_{G}^{1}(P)$, the space of right-invariant 1-forms on $P$.

We identify the cotangent space $T_{\varphi}^{*} \mathcal{G} a u(P)$ with the tangent space $T_{\varphi} \mathcal{G} a u(P)$ via the duality

$$
\left\langle U_{\varphi}, V_{\varphi}\right\rangle:=\int_{M} \gamma\left(\widetilde{\mathcal{A}\left(U_{\varphi}\right)}, \widetilde{\mathcal{A}\left(V_{\varphi}\right)}\right) \mu
$$

for any principal connection $\mathcal{A}$ on $P$. Note that, since $U_{\varphi}$ and $V_{\varphi}$ are vertical, the pairing (2.5) does not depend on $\mathcal{A}$, indeed, we have $\mathcal{A}\left(U_{\varphi}\right)=\sigma^{-1}\left(U_{\varphi} \circ \varphi^{-1}\right) \circ \varphi$.

\section{Equations for the fields}

In this section we give the Hamiltonian formulation for the Yang-Mills fields in the vacuum. We first treat the electromagnetic case.

3.1. Hamiltonian formulation of the Maxwell equations. The Hamiltonian is defined on the cotangent bundle $T^{*} \Omega^{1}(M, \mathbb{R})$ and is given by

$$
H(A, Y)=\frac{1}{2} \int_{M}\|E\|^{2} \mu+\frac{1}{2} \int_{M}\|B\|^{2} \mu,
$$

where $E:=-Y$ is the electric field and $B:=\mathbf{d} A$ is the magnetic field. Hamilton's equations are

$$
\frac{\partial B}{\partial t}=-\mathbf{d} E \text { and } \quad \frac{\partial E}{\partial t}=\delta B
$$

and the relation $B=\mathbf{d} A$ gives

$$
\mathbf{d} B=0 .
$$

To obtain the last equation $\delta E=0$ we use the invariance of the Hamiltonian under gauge transformations. The action of the gauge group $\mathcal{F}(M)$ on $\Omega^{1}(M, \mathbb{R})$ is given by

$$
\mathcal{F}(M) \times \Omega^{1}(M, \mathbb{R}) \rightarrow \Omega^{1}(M, \mathbb{R}), \quad(\varphi, A) \mapsto A+\mathbf{d} \varphi,
$$

and is Hamiltonian. The associated momentum map is

$$
\mathbf{J}: T^{*} \Omega^{1}(M, \mathbb{R}) \rightarrow \mathcal{F}(M)^{*}, \quad \mathbf{J}(A, Y)=\delta Y,
$$


so the condition $\mathbf{J}(A, Y)=0$ gives the Gauss law

$$
\delta E=0 .
$$

Remark that we identify the tangent and cotangent spaces of $\Omega^{1}(M, \mathbb{R})$ and $\mathcal{F}(M)$ using the natural $L^{2}$-pairing.

When $M$ is three dimensional, we can define the vector fields $\mathbf{E}:=E^{\sharp}$ and $\mathbf{B}:=(\star B)^{\sharp}$. In this case, equations (3.1), (3.2) and (3.4) are equivalent to the Maxwell equations in the vacuum

$$
\begin{cases}\frac{\partial \mathbf{E}}{\partial t}=\operatorname{curl} \mathbf{B}, & \operatorname{div} \mathbf{E}=0 \\ \frac{\partial \mathbf{B}}{\partial t}=-\operatorname{curl} \mathbf{E}, & \operatorname{div} \mathbf{B}=0\end{cases}
$$

3.2. Generalization to any principal bundle. We now generalize the previous formulation to the case of a $G$-principal bundle $P \rightarrow M$ over an arbitrary compact boundaryless manifold $M$. When $P$ is a trivial $S^{1}$ bundle over a three dimensional manifold, we recover the Maxwell equations.

As in the electromagnetic case, the configuration space variable is the magnetic potential $\mathcal{A} \in \mathcal{C} \operatorname{Conn}(P)$ and the Hamiltonian is defined on the cotangent bundle $T^{*} \operatorname{Conn}(P)$ by

$$
H(\mathcal{A}, \mathcal{Y})=\frac{1}{2} \int_{M}\|E\|^{2} \mu+\frac{1}{2} \int_{M}\|B\|^{2} \mu
$$

where:

(1) $E:=\widetilde{\mathcal{E}} \in \Omega^{1}(M, \operatorname{Ad} P)$ is the $\operatorname{Ad} P$-valued 1-form associated, through the map (2.1), to the "electric part" $\mathcal{E} \in \overline{\Omega^{1}}(P, \mathfrak{g})$ of the Yang-Mills field, given by

$$
\mathcal{E}:=-\mathcal{Y} \in \overline{\Omega^{1}}(P, \mathfrak{g}),
$$

(2) $B:=\widetilde{\mathcal{B}} \in \Omega^{2}(M, \operatorname{Ad} P)$ is the $\operatorname{Ad} P$-valued 2-form associated to the "magnetic part" $\mathcal{B} \in \overline{\Omega^{1}}(P, \mathfrak{g})$ of the Yang-Mills field, given by the curvature

$$
\mathcal{B}:=\mathbf{d}^{\mathcal{A}} \mathcal{A} \in \overline{\Omega^{2}}(P, \mathfrak{g}) .
$$

As before, we identify the cotangent bundle of $\mathcal{C}$ onn $(P)$ with the tangent bundle, using the $L^{2}$ pairing

$$
\langle\alpha, \beta\rangle=\int_{M}(g \gamma)(\alpha, \beta) \mu, \quad \alpha, \beta \in \Omega^{k}(M, \operatorname{Ad} P) .
$$

The Hamiltonian equations associated to $H$ are

$$
\frac{\partial \mathcal{B}}{\partial t}=-\mathbf{d}^{\mathcal{A}} \mathcal{E} \quad \text { and } \quad \frac{\partial \mathcal{E}}{\partial t}=\delta^{\mathcal{A}} \mathcal{B},
$$

and the Bianchi identity gives

$$
\mathbf{d}^{\mathcal{A}} \mathcal{B}=0 .
$$

To obtain the last equation we use the invariance of the Hamiltonian under the gauge transformations. The action of $\varphi \in \mathcal{G} a u(P)$ on $\mathcal{A} \in \mathcal{C}$ onn $(P)$ is given by $\varphi^{*} \mathcal{A}$ and the cotangent lift of this action is given by $\left(\varphi^{*} \mathcal{A}, \varphi^{*} \mathcal{Y}\right)$. Under this action, $\mathcal{E}$ and $\mathcal{B}$ are transformed into $\varphi^{*} \mathcal{E}$ and $\varphi^{*} \mathcal{B}$, so $H$ is gauge-invariant. The momentum mapping associated to this Hamiltonian action is given by

$$
\mathbf{J}: T^{*} \operatorname{Conn}(P) \rightarrow \mathfrak{g a u}(P)^{*}, \quad \mathbf{J}(\mathcal{A}, \mathcal{Y})=\sigma\left(\delta^{\mathcal{A}} \mathcal{Y}\right),
$$


so the conservation law $\mathbf{J}(\mathcal{A}, \mathcal{Y})=0$ gives the last equation

$$
\delta^{\mathcal{A}} \mathcal{E}=0 .
$$

Note that we identify $\mathfrak{g a u}(P)^{*}$ with $\mathfrak{g a \mathfrak { u }}(P)$ via the $L^{2}$ pairing (3.6).

\section{Hamiltonian formulation of Euler-Yang-Mills}

We begin by quickly recalling some facts about the Hamiltonian semidirect product reduction theory.

Hamiltonian semidirect product reduction with parameter. Let $\rho$ : $G \rightarrow \operatorname{Aut}(V)$ denote a right Lie group representation of $G$ in the vector space $V$. As sets, the semidirect product $S=G(S)$ is the Cartesian product $S=G \times V$ whose group multiplication is given by

$$
\left(g_{1}, v_{1}\right)\left(g_{2}, v_{2}\right)=\left(g_{1} g_{2}, v_{2}+\rho_{g_{2}}\left(v_{1}\right)\right) .
$$

Below we shall use the operation $\diamond: V \times V^{*} \rightarrow \mathfrak{g}^{*}$ defined by

$$
\langle v \diamond a, \xi\rangle_{\mathfrak{g}}:=-\langle a \xi, v\rangle_{V},
$$

where $v \in V, a \xi \in V^{*}$ denotes the induced $\mathfrak{g}$-action of $\xi \in \mathfrak{g}$ on $a \in V^{*}$, and $\langle\cdot, \cdot\rangle_{\mathfrak{g}}: \mathfrak{g}^{*} \times \mathfrak{g} \rightarrow \mathbb{R}$ and $\langle\cdot, \cdot\rangle_{V}: V^{*} \times V \rightarrow \mathbb{R}$ are the duality parings.

The lift of right translation of $S$ on $T^{*} S$ induces a right action on $T^{*} G \times V^{*}$. Let $Q$ be another manifold (without any $G$ or $V$-action). Consider a Hamiltonian function $H: T^{*} G \times T^{*} Q \times V^{*} \rightarrow \mathbb{R}$ right invariant under the $S$-action on $T^{*} G \times$ $T^{*} Q \times V^{*}$; recall that the $S$-action on $T^{*} Q$ is trivial. In particular, the function $H_{a_{0}}:=\left.H\right|_{T^{*} G \times T^{*} Q \times\left\{a_{0}\right\}}: T^{*} G \times T^{*} Q \rightarrow \mathbb{R}$ is invariant under the induced action of the isotropy subgroup $G_{a_{0}}:=\left\{g \in G \mid \rho_{g}^{*} a_{0}=a_{0}\right\}$ for any $a_{0} \in V^{*}$. The following theorem is an easy consequence of the semidirect product reduction theorem (see $[\mathbf{M R W}]$ ) and the reduction by stages method (see [MMOPR]).

Theorem 4.1. For $\alpha(t) \in T_{g(t)}^{*} G$ and $\mu(t):=T^{*} R_{g(t)}(\alpha(t)) \in \mathfrak{g}^{*}$, the following are equivalent:

i $(\alpha(t), q(t), p(t))$ satisfies Hamilton's equations for $H_{a_{0}}$ on $T^{*}(G \times Q)$.

ii The following system of Lie-Poisson equations with parameter coupled with Hamilton's equations holds on $\mathfrak{s}^{*} \times T^{*} Q$ :

$$
\frac{\partial}{\partial t}(\mu, a)=-\operatorname{ad}_{\left(\frac{\delta h}{\delta \mu}, \frac{\delta h}{\delta a}\right)}^{*}(\mu, a)=-\left(\operatorname{ad}_{\frac{\delta h}{\delta \mu}}^{*} \mu+\frac{\delta h}{\delta a} \diamond a, a \frac{\delta h}{\delta \mu}\right), \quad a(0)=a_{0}
$$

and

$$
\frac{d q^{i}}{d t}=\frac{\partial h}{\partial p_{i}}, \quad \frac{d p_{i}}{d t}=-\frac{\partial h}{\partial q^{i}},
$$

where $\mathfrak{s}$ is the semidirect product Lie algebra $\mathfrak{s}=\mathfrak{g}(5)$. The associated Poisson bracket is the sum of the Lie-Poisson bracket on the Lie algebra $\mathfrak{s}^{*}$ and the canonical bracket on the cotangent bundle $T^{*} Q$

$$
\{f, g\}(\mu, a, q, p)=\left\langle\mu,\left[\frac{\delta f}{\delta \mu}, \frac{\delta g}{\delta \mu}\right]\right\rangle+\left\langle a, \frac{\delta f}{\delta a} \frac{\delta g}{\delta \mu}-\frac{\delta g}{\delta a} \frac{\delta f}{\delta \mu}\right\rangle+\frac{\partial f}{\partial q^{i}} \frac{\partial g}{\partial p_{i}}-\frac{\partial g}{\partial q^{i}} \frac{\partial f}{\partial p_{i}} .
$$


The Hamiltonian. The Hamiltonian for the Euler-Yang-Mills equations is defined on the cotangent bundle $T^{*}(\mathcal{A u t}(P) \times \mathcal{C}$ onn $(P))$ and is given, for $(\rho, \sigma) \in$ $\mathcal{F}(M)^{* 2}$, by

$$
\begin{aligned}
H_{(\rho, \sigma)}\left(\mathbf{M}_{\psi}, \mathcal{A}, \mathcal{Y}\right)= & \frac{1}{2} \int_{M} \frac{1}{\rho} K_{\mathcal{A}}^{*}\left(\mathbf{M}_{\psi}, \mathbf{M}_{\psi}\right) \mu+\int_{M} \rho e\left(\rho(J \bar{\psi})^{-1}, \sigma\right) \mu \\
& +\frac{1}{2} \int_{M}\|E\|^{2} \mu+\frac{1}{2} \int_{M}\|B\|^{2} \mu,
\end{aligned}
$$

where $e$ is the fluid's specific internal energy and $K_{\mathcal{A}}^{*}$ is the metric induced on $T^{*} P$ by $K_{\mathcal{A}}$. This Hamiltonian is obtained by integrating the Kaluza-Klein energy of the particles and by summing it with the internal energy of the fluid and the Hamiltonian for the Yang-Mills field. By Theorem 4.1, Hamilton's equations for $H_{(\rho, \sigma)}$ are equivalent to the Lie-Poisson equations on the dual of the semidirect product Lie algebra $\mathfrak{a u t}(P) \subseteq \mathcal{F}(M)^{2}$, together with the Hamilton equations on $T^{*} \mathcal{C} \operatorname{onn}(P)$, relative to the reduced Hamiltonian $h$ given on $\left(\mathfrak{a u t}(P)(S) \mathcal{F}(M)^{2}\right)^{*} \times$ $T^{*} \operatorname{Conn}(P)$ by

$h(\mathbf{M}, \rho, \sigma, \mathcal{A}, \mathcal{Y})=\frac{1}{2} \int_{M} \frac{1}{\rho} K_{\mathcal{A}}^{*}(\mathbf{M}, \mathbf{M}) \mu+\int_{M} \rho e(\rho, \sigma) \mu+\frac{1}{2} \int_{M}\|E\|^{2} \mu+\frac{1}{2} \int_{M}\|B\|^{2} \mu$.

The momentum map of the gauge group. As it was the case in section 3 , the nonabelian Gauss equation

$$
\delta^{\mathcal{A}} \mathcal{E}=-\mathcal{Q}
$$

is obtained by invariance of the Hamiltonian under the gauge transformations. Indeed, consider the action of the gauge group given for $\eta \in \mathcal{G} a u(P)$, by

$$
(\psi, \mathcal{A}) \mapsto\left(\eta^{-1} \circ \psi, \eta^{*} \mathcal{A}\right)
$$

The cotangent-lift of this action leaves the Hamiltonian invariant. So the associated momentum map, which is computed in the following lemma, is a conserved quantity.

LEMMA 4.2. The momentum mapping associated to the cotangent lift of the gauge group action is given by

$$
\boldsymbol{J}\left(\mathbf{M}_{\psi}, \mathcal{A}, \mathcal{Y}\right)=\sigma\left(\delta^{\mathcal{A}} \mathcal{Y}-\mathcal{A}\left(\left(J \bar{\psi}^{-1}\right) V_{\psi} \circ \psi^{-1}\right)\right) \in \mathfrak{g a u}(P)^{*},
$$

where $V_{\psi} \in T_{\psi} \mathcal{A}$ ut $(P)$ is such that $\mathbf{M}_{\psi}=K_{\mathcal{A}}\left(V_{\psi}, \cdot\right)$ and $\sigma: \mathcal{F}_{G}(P, \mathfrak{g}) \rightarrow \mathfrak{g a u}(P)$ is defined in (2.4).

When $\mathbf{M}_{\psi}=K_{\mathcal{A}}\left(\rho_{0} U_{\psi}, \cdot\right)$, is a solution of Hamilton's equations associated to $H_{\left(\rho_{0}, \sigma_{0}\right)}$, the conservation law $\mathbf{J}\left(\mathbf{M}_{\psi}, \mathcal{A}, \mathcal{Y}\right)=0$ gives

$$
\mathcal{A}\left(\left(J \bar{\psi}^{-1}\right)\left(\rho_{0} \circ \bar{\psi}^{-1}\right) U_{\psi} \circ \psi^{-1}\right)=\delta^{\mathcal{A}} \mathcal{Y} .
$$

Using the definition of the charge density $\mathcal{Q}:=\rho \mathcal{A}(U)$, the identities $U_{\psi} \circ \psi^{-1}=U$, $\left(J \bar{\psi}^{-1}\right)\left(\rho_{0} \circ \bar{\psi}^{-1}\right)=\rho$, and the notation $\mathcal{E}=-\mathcal{Y}$, we get

$$
\mathcal{Q}=-\delta^{\mathcal{A}} \mathcal{E}
$$

We can now state our main result. 
Theorem 4.3. Let $\left(\mathbf{M}_{\psi}, \mathcal{A}, \mathcal{Y}\right)$ be a curve in the cotangent bundle $T^{*}(\mathcal{A} u t(P) \times$ $\mathcal{C}$ onn $(P))$, and consider the induced curve $(\mathbf{M}, \mathcal{A}, \mathcal{Y}) \in \mathfrak{a u t}(P)^{*} \times T^{*} \mathcal{C}$ onn $(P)$ given by $\mathbf{M}:=(J \bar{\psi}) \mathbf{M}_{\psi} \circ \psi^{-1}$. Then $\left(\mathbf{M}_{\psi}, \mathcal{A}, \mathcal{Y}\right)$ is a solution of the Hamilton's equations associated to the Hamiltonian $H_{\left(\rho_{0}, \sigma_{0}\right)}$ given in (4.1) if and only if $(\mathbf{M}, \mathcal{A}, \mathcal{Y})$ is a solution of the system

$$
\left\{\begin{array}{l}
\frac{\partial v}{\partial t}+\nabla_{v} v=\frac{1}{\rho} \gamma(Q, E(\cdot)+B(\cdot, v))^{\sharp}-\frac{1}{\rho} \operatorname{grad} p \\
\frac{\partial \rho}{\partial t}+\operatorname{div}(\rho v)=0, \rho(0)=\rho_{0}, \quad \frac{\partial \sigma}{\partial t}+\mathbf{d} \sigma(v)=0, \sigma(0)=\sigma_{0} \\
\frac{\partial Q}{\partial t}+\nabla_{v}^{\mathcal{A}} Q+Q \operatorname{div} v=0 \\
\frac{\partial \mathcal{E}}{\partial t}=\delta^{\mathcal{A}} \mathcal{B}-Q v^{b} \\
\frac{\partial \mathcal{B}}{\partial t}=-\mathbf{d}^{\mathcal{A}} \mathcal{E}
\end{array}\right.
$$

where

$$
\begin{aligned}
p & :=\rho^{2} \frac{\partial e}{\partial \rho}(\rho, \sigma), \\
\mathcal{E} & :=-\mathcal{Y} \in \overline{\Omega^{1}}(P, \mathfrak{g}) \quad \text { and } \quad E:=\widetilde{\mathcal{E}} \in \Omega^{1}(M, \operatorname{Ad} P), \\
\mathcal{B}: & =\mathbf{d}^{\mathcal{A}} \mathcal{A} \quad \text { and } \quad B:=\widetilde{\mathcal{B}}, \\
Q & :=\widetilde{\mathcal{Q}} \in \Gamma(\operatorname{Ad} P) .
\end{aligned}
$$

The Eulerian velocity $v$ and the gauge-charge density $\mathcal{Q}$ are given in terms of the momentum $\mathbf{M}$ by

$$
v=[U] \quad \text { and } \quad \mathcal{Q}=\mathcal{A}(\rho U) \quad \text { where } U=K_{\mathcal{A}}^{*}\left(\frac{\mathbf{M}}{\rho}, \cdot\right) .
$$

Conservation of the momentum map associated to the gauge transformations gives the equation

$$
\delta^{\mathcal{A}} \mathcal{E}=-\mathcal{Q} .
$$

Thus we have recovered the Euler-Yang-Mills equations as given in [GHK]. One can adapt this theorem to the incompressible and homogeneous case.

Corollary 4.4. In the Abelian case of the trivial bundle $P=M \times S^{1}$ and assuming that the fluid is composed of particles of mass $m$ and charge $q$, we obtain the Euler-Maxwell equations

$$
\left\{\begin{array}{l}
\frac{\partial v}{\partial t}+\nabla_{v} v=\frac{q}{m}(\mathbf{E}+v \times \mathbf{B})-\frac{1}{\rho} \operatorname{grad} p \\
\frac{\partial \rho}{\partial t}+\operatorname{div}(\rho v)=0, \rho(0)=\rho_{0}, \quad \frac{\partial \sigma}{\partial t}+\mathbf{d} \sigma(v)=0, \sigma(0)=\sigma_{0} \\
\frac{\partial \mathbf{E}}{\partial t}=\operatorname{curl} \mathbf{B}-\frac{q}{m} \rho v, \quad \frac{\partial \mathbf{B}}{\partial t}=-\operatorname{curl} \mathbf{E} \\
\operatorname{div} \mathbf{E}=\frac{q}{m} \rho, \quad \operatorname{div} \mathbf{B}=0
\end{array}\right.
$$

where

$$
\mathbf{E}:=E^{\sharp} \quad \text { and } \quad \mathbf{B}:=(\star B)^{\sharp} \text {. }
$$


Proof. If we define $Q_{t}=\rho_{t} \frac{q_{t}}{m}$, the equation for $Q$ in (4.3) becomes

$$
0=\frac{\partial q_{t}}{\partial t}+\mathbf{d} q_{t}(v)=\frac{d}{d t} q_{t}(x(t))
$$

where $x(t)$ is the trajectory of the particle starting at $x(0)$. Since all particles have the same charge $q \in \mathbb{R}$ by hypothesis, we conclude that $q(t, x)$ is a constant. Therefore, the equation for $Q$ in (4.3) disappears. It is easily seen that the other equations become the ones in (4.5).

Poisson bracket. From Theorem 4.1 we know that the Euler-Yang-Mills equations can be written

$$
\dot{f}=\{f, h\}
$$

with respect to the Poisson bracket

$$
\begin{aligned}
\{f, g\}(\mathbf{M}, \rho, \sigma, \mathcal{A}, \mathcal{Y}) & =\int_{M} \mathbf{M}\left(\left[\frac{\delta f}{\delta \mathbf{M}}, \frac{\delta g}{\delta \mathbf{M}}\right]_{L}\right) \mu \\
& +\int_{M} \rho\left(\mathbf{d}\left(\frac{\delta f}{\delta \rho}\right)\left[\frac{\delta g}{\delta \mathbf{M}}\right]-\mathbf{d}\left(\frac{\delta g}{\delta \rho}\right)\left[\frac{\delta f}{\delta \mathbf{M}}\right]\right) \mu \\
& +\int_{M} \sigma\left(\operatorname{div}\left(\frac{\delta f}{\delta \sigma}\left[\frac{\delta g}{\delta \mathbf{M}}\right]\right)-\operatorname{div}\left(\frac{\delta g}{\delta \sigma}\left[\frac{\delta f}{\delta \mathbf{M}}\right]\right)\right) \mu \\
& +\int_{M}(g \gamma)\left(\frac{\delta f}{\delta \mathcal{A}}, \frac{\delta g}{\delta \mathcal{Y}}\right) \mu-\int_{M}(g \gamma)\left(\frac{\delta g}{\delta \mathcal{A}}, \frac{\delta f}{\delta \mathcal{Y}}\right) \mu .
\end{aligned}
$$

We can obtain this bracket and the associated Hamilton equations (4.3) alternatively by a reduction by stages process (see [MMOPR]). The symplectic reduced spaces are of the form $\mathcal{O} \times T^{*} \mathcal{C o n n}(P)$, where $\mathcal{O}$ is a coadjoint orbit of the semidirect product $S:=\mathcal{A} u t(P) \subseteq(\mathcal{F}(M) \times \mathcal{F}(M))$.

If the principal bundle is trivial, the automorphism group is a semidirect product of groups. In this case the first term can be written more explicitly by taking advantage of the internal structure of $\mathcal{A} u t(P)$, and we recover (up to sign conventions) the Poisson bracket given in equation (38) in [GHK].

The second reduction. Note that right translation of $\mathcal{A} u t(P) \subseteq(\mathcal{F}(M) \times$ $\mathcal{F}(M)$ ) on itself and the action of $\mathcal{G} a u(P)$ on $\mathcal{A u t}(P) \times \mathcal{C}$ onn $(P)$ given by (4.2) commute if one views them as actions on $S \times \mathcal{C}$ onn $(P)$. Therefore, by the general theory of commuting reduction by stages (see [MMOPR]), the momentum map associated to the gauge group action is $\mathcal{A} u t(P)$-invariant and induces a momentum map $\mathbf{J}_{\mathfrak{s}^{*}}$ on $\mathfrak{s}^{*} \times T^{*} \mathcal{C}$ onn $(P)$ which restricts to a momentum map $\mathbf{J}_{\mathcal{O}}$ on the reduced space $\mathcal{O} \times T^{*} \operatorname{Conn}(P)$. A direct computation shows that we have

$$
\mathbf{J}_{\mathfrak{s}^{*}}: \mathfrak{s}^{*} \times T^{*} \operatorname{Conn}(P) \rightarrow \mathfrak{g a u}(P)^{*}, \quad \mathbf{J}_{\mathfrak{s}}^{*}(\mathbf{M}, \rho, \sigma, \mathcal{A}, \mathcal{Y})=\sigma\left(\delta^{\mathcal{A}} \mathcal{Y}-\mathcal{A}(V)\right),
$$

where $V \in \mathfrak{a u t}(P)$ is such that $\mathbf{M}=K_{\mathcal{A}}(V, \cdot)$, and $\sigma$ on the right hand side denotes the map defined in (2.4). The gauge group action induced on $\mathfrak{s}^{*} \times T^{*} \operatorname{Conn}(P)$ and $\mathcal{O} \times T^{*} \operatorname{Conn}(P)$ is given by

$$
(\mathbf{M}, \rho, \sigma, \mathcal{A}, \mathcal{Y}) \mapsto\left(\operatorname{Ad}_{\eta}^{*} \mathbf{M}, \rho, \sigma, \eta^{*} \mathcal{A}, \eta^{*} \mathcal{Y}\right) .
$$

Using the notation $\mathbf{S}:=(\mathbf{M}, \rho, \sigma) \in \mathcal{O}$, it can be written as

$$
(\mathbf{S}, \mathcal{A}, \mathcal{Y}) \mapsto\left(\operatorname{Ad}_{(\eta, 0,0)}^{*} \mathbf{S}, \eta^{*} \mathcal{A}, \eta^{*} \mathcal{Y}\right) .
$$

This action is simply the diagonal action given on the first factor by the coadjoint action of the subgroup $\mathcal{G} a u(P)$ of $S=\mathcal{A} u t(P)(S(\mathcal{F}(M) \times \mathcal{F}(M))$, and on the 
second factor by the gauge transformations. Note that when the center $Z(G)$ of the structure group $G$ of the bundle $\pi: P \rightarrow M$ is trivial, then the transformation $\mathcal{A} \mapsto \eta^{*} \mathcal{A}$ is free. In this case, the reduced action (4.9) is also free and the second reduced symplectic spaces

$$
\mathbf{J}_{\mathcal{O}}^{-1}(\mathbf{N}) / \mathcal{G} a u(P)_{\mathbf{N}}, \quad \mathbf{N} \in \mathfrak{g a u}(P)^{*},
$$

have no singularities.

By the reduction by stages process (see [MMOPR]), the reduced spaces $\mathbf{J}_{\mathcal{O}}^{-1}(\mathbf{N}) / \mathcal{G} a u(P)_{\mathbf{N}}$ are symplectically diffeomorphic to the reduced spaces obtained by a one step reduction from the cotangent bundle

$$
T^{*}(S \times \mathcal{C} \text { onn }(P))
$$

with respect to the product of the two cotangent-lifted actions.

Note that the Euler-Yang-Mills equation (4.3) projects to the reduced space at zero momentum

$$
\mathbf{J}_{\mathcal{O}}^{-1}(0) / \mathcal{G} a u(P)
$$

The general case corresponds to the Yang-Mills fluid with an external charge $\mathbf{N}$.

In order to obtain the reduced Poisson structure concretely, we will identify the space $\mathfrak{s}^{*} \times T^{*} \mathcal{C} \operatorname{onn}(P)$ with a space on which the gauge action is simpler. This identification is given in the following proposition.

Proposition 4.5. Consider the group $K=\mathcal{D}(M) \subseteq(\mathcal{F}(M) \times \mathcal{F}(M))$ and denote by $\mathfrak{k}^{*}$ the dual of its Lie algebra. There is a gauge-equivariant diffeomorphism

$$
i: \mathfrak{s}^{*} \times T^{*} \operatorname{Conn}(P) \rightarrow \mathfrak{k}^{*} \times \mathcal{F}_{G}\left(P, \mathfrak{g}^{*}\right) \times T^{*} \operatorname{Conn}(P),
$$

given by

$$
i(\mathbf{M}, \rho, \sigma, \mathcal{A}, \mathcal{Y}):=\left(\left(\operatorname{Hor}_{\mathcal{A}}\right)^{*} \circ \mathbf{M}, \rho, \sigma, \mathbb{J} \circ \mathbf{M}, \mathcal{A},-\mathcal{Y}\right)=:(\mathbf{n}, \rho, \sigma, \nu, \mathcal{A}, \mathcal{E}),
$$

where the gauge group acts on $\mathfrak{s}^{*} \times T^{*} \operatorname{Conn}(P)$ by the action (4.9) and on $\mathfrak{k}^{*} \times$ $\mathcal{F}_{G}\left(P, \mathfrak{g}^{*}\right) \times T^{*} \mathcal{C}$ onn $(P)$ only on the factor $\mathcal{F}_{G}\left(P, \mathfrak{g}^{*}\right) \times T^{*} \mathcal{C}$ onn $(P)$ by the right action

$$
(\nu, \mathcal{A}, \mathcal{E}) \mapsto\left(\nu \circ \eta, \eta^{*} \mathcal{A}, \eta^{*} \mathcal{E}\right) .
$$

Moreover, the image of the level set $\mathbf{J}_{\mathfrak{5}^{*}}^{-1}(\mathbf{N})$ by the diffeomorphism $i$ is given by

$$
\left\{(\mathbf{n}, \rho, \sigma, \nu, \mathcal{A}, \mathcal{E}) \mid \nu+\gamma\left(\delta^{\mathcal{A}} \mathcal{E}+f, \cdot\right)=0\right\},
$$

where $\mathbf{N} \in \mathfrak{g a u}(P)$ and $f \in \mathcal{F}_{G}(P, \mathfrak{g})$ is such that $\sigma(f)=\mathbf{N}$. Thus $\mathbf{J}_{\mathfrak{s}^{*}}^{-1}(\mathbf{N})$ is diffeomorphic to $\mathfrak{k}^{*} \times T^{*} \operatorname{Conn}(P)$.

The map $\mathbb{J}: T^{*} P \rightarrow \mathfrak{g}^{*}$ denotes the momentum map $\mathbb{J}\left(\alpha_{p}\right)(\xi):=\left\langle\alpha_{p}, \xi_{P}(p)\right\rangle$, and $\left(\operatorname{Hor}_{\mathcal{A}}\right)^{*}$ denotes the dual map of the horizontal-lift $\operatorname{Hor}_{\mathcal{A}}: T M \rightarrow T P$ with respect to $\mathcal{A}$.

From this Proposition we obtain that the reduced spaces $\mathbf{J}_{\mathfrak{S}^{*}}^{-1}(\mathbf{N}) / \mathcal{G} a u(P)_{\mathbf{N}}$ can be identified with the quotient space $\mathfrak{k}^{*} \times\left[\left(\mathcal{F}_{G}\left(P, \mathfrak{g}^{*}\right) \times T^{*} \operatorname{Conn}(P)\right) / \mathcal{G} a u(P)_{\mathbf{N}}\right]$, via the diffeomorphism induced by $i$ and given by

$$
[(\mathbf{M}, \rho, \sigma, \nu, \mathcal{A}, \mathcal{Y})] \mapsto\left(\left(\operatorname{Hor}_{\mathcal{A}}\right)^{*} \circ \mathbf{M}, \rho, \sigma,[\nu, \mathcal{A}, \mathcal{E}]\right),
$$

where [.] denote the corresponding equivalence classes. 
We now compute the Poisson structure $\{,\}^{\prime}$ induced by $i$ on $\mathfrak{k}^{*} \times \mathcal{F}_{G}\left(P, \mathfrak{g}^{*}\right) \times$ $T^{*} \mathcal{C} \operatorname{onn}(P)$. For $f, g \in \mathcal{F}\left(\mathfrak{k}^{*} \times \mathcal{F}_{G}\left(P, \mathfrak{g}^{*}\right) \times T^{*} \mathcal{C} \operatorname{onn}(P)\right)$ we have the formulas

$$
\begin{aligned}
& \frac{\delta(f \circ i)}{\delta \mathbf{M}}=\operatorname{Hor}_{\mathcal{A}} \circ \frac{\delta f}{\delta \mathbf{n}}+J^{*} \circ \frac{\delta f}{\delta \nu}, \quad\left[\frac{\delta(f \circ i)}{\delta \mathbf{M}}\right]=\frac{\delta f}{\delta \mathbf{n}}, \quad \mathbf{n}:=\left(\operatorname{Hor}_{\mathcal{A}}\right)^{*} \circ \mathbf{M}, \\
& (g \gamma)\left(\frac{\delta(f \circ i)}{\delta \mathcal{A}}, \mathcal{C}\right)=(g \gamma)\left(\frac{\delta f}{\delta \mathcal{A}}, \mathcal{C}\right)-\nu\left(\mathcal{C}\left(\operatorname{Hor}_{\mathcal{A}}\left(\frac{\delta f}{\delta \mathbf{n}}\right)\right)\right), \quad \nu:=\mathbf{J} \circ \mathbf{M} .
\end{aligned}
$$

Therefore, we obtain

$$
\begin{aligned}
&\{f, g\}^{\prime}(\mathbf{n}, \rho, \sigma, \nu, \mathcal{A}, \mathcal{E}):=\{f \circ i, g \circ i\}(\mathbf{M}, \rho, \sigma, \mathcal{A}, \mathcal{Y}) \\
&=\int_{M} \mathbf{n}\left(\left[\frac{\delta f}{\delta \mathbf{n}}, \frac{\delta g}{\delta \mathbf{n}}\right]_{L}\right) \mu+\int_{M} \rho\left(\mathbf{d}\left(\frac{\delta f}{\delta \rho}\right) \frac{\delta g}{\delta \mathbf{n}}-\mathbf{d}\left(\frac{\delta g}{\delta \rho}\right) \frac{\delta f}{\delta \mathbf{n}}\right) \mu \\
&+ \int_{M} \sigma\left(\operatorname{div}\left(\frac{\delta f}{\delta \sigma} \frac{\delta g}{\delta \mathbf{n}}\right)-\operatorname{div}\left(\frac{\delta g}{\delta \sigma} \frac{\delta f}{\delta \mathbf{n}}\right)\right) \mu \\
&+ \int_{M}(g \gamma)\left(\frac{\delta g}{\delta \mathcal{A}}, \frac{\delta f}{\delta \mathcal{E}}\right) \mu-\int_{M}(g \gamma)\left(\frac{\delta f}{\delta \mathcal{A}}, \frac{\delta g}{\delta \mathcal{E}}\right) \mu \\
&+ \int_{M} \nu\left(\left[\frac{\delta f}{\delta \nu}, \frac{\delta g}{\delta \nu}\right]\right) \mu \\
&+ \int_{M} \nu\left(\frac{\delta g}{\delta \mathcal{E}}\left(\operatorname{Hor}_{\mathcal{A}} \circ \frac{\delta f}{\delta \mathbf{n}}\right)-\frac{\delta f}{\delta \mathcal{E}}\left(\operatorname{Hor}_{\mathcal{A}} \circ \frac{\delta g}{\delta \mathbf{n}}\right)\right. \\
&+\mathbf{d}^{\mathcal{A}}\left(\frac{\delta f}{\delta \nu}\right)\left(\operatorname{Hor}_{\mathcal{A}} \circ \frac{\delta g}{\delta \mathbf{n}}\right)-\mathbf{d}^{\mathcal{A}}\left(\frac{\delta g}{\delta \nu}\right)\left(\operatorname{Hor}_{\mathcal{A}} \circ \frac{\delta f}{\delta \mathbf{n}}\right) \\
&\left.+\mathcal{B}\left(\operatorname{Hor}_{\mathcal{A}} \circ \frac{\delta f}{\delta \mathbf{n}}, \operatorname{Hor}_{\mathcal{A}} \circ \frac{\delta g}{\delta \mathbf{n}}\right)\right) \mu .
\end{aligned}
$$

Note that the first three terms in (4.14) represent the Lie-Poisson bracket on $\mathfrak{k}^{*}$, the fourth and fifth terms represent the canonical bracket on $T^{*} \mathcal{C} o n n(P)$, the sixth term is the Lie-Poisson bracket on $\mathcal{F}_{G}\left(P, \mathfrak{g}^{*}\right)$, and the last term provides the coupling of the fluid variables to the Yang-Mills fields.

By the general process of Poisson (point) reduction, the reduced spaces

$$
\mathbf{J}_{\mathfrak{s}^{*}}^{-1}(\mathbf{N}) / \mathcal{G} a u(P)_{\mathbf{N}} \simeq \mathfrak{k}^{*} \times\left[\left(\mathcal{F}_{G}\left(P, \mathfrak{g}^{*}\right) \times T^{*} \operatorname{Conn}(P)\right) / \mathcal{G} a u(P)_{\mathbf{N}}\right]
$$

inherit a Poisson bracket $\{,\}_{\mathbf{N}}$ given by

$$
\left\{f_{\mathbf{N}}, g_{\mathbf{N}}\right\}_{\mathbf{N}}(\mathbf{n}, \rho, \sigma,[\nu, \mathcal{A}, \mathcal{E}]):=\{f, g\}^{\prime}(\mathbf{n}, \rho, \sigma, \nu, \mathcal{A}, \mathcal{Y}),
$$

where $f, g$ are any $\mathcal{G} a u(P)$-invariant extensions of the functions $f_{\mathbf{N}} \circ \pi_{\mathbf{N}}, g_{\mathbf{N}} \circ \pi_{\mathbf{N}}$ : $\mathbf{J}_{\mathfrak{s}^{*}}^{-1}(\mathbf{N}) \rightarrow \mathbb{R}$, relative to the projection $\pi_{\mathbf{N}}: \mathbf{J}_{\mathfrak{s}^{*}}^{-1}(\mathbf{N}) \rightarrow \mathbf{J}_{\mathfrak{s}^{*}}^{-1}(\mathbf{N}) / \mathcal{G} a u(P)_{\mathbf{N}}$.

The equations of motion on the Poisson point reduced space $\mathbf{J}_{\mathcal{O}}^{-1}(0) / \mathcal{G} a u(P)$ cannot be written explicitly, because there is no concrete realization of this quotient, to our knowledge. However, in the particular case of a trivial principal bundle, this is possible.

We end this section by examining the case of the trivial bundle $P=S^{1} \times M$, that is, the case of the Euler-Maxwell equations. Since $G=S^{1}$, the gauge transformation (4.8) is simply

$$
(\mathbf{m}, \nu, \rho, \sigma, A, Y) \mapsto(\mathbf{m}+\nu \mathbf{d} \eta, \nu, \rho, \sigma, A+\mathbf{d} \eta, Y),
$$


where $\nu=Q=\rho \frac{q}{m}$, by relation (4.4) and Corollary 4.4. This gauge transformation coincides with the one given in equation (36) in [MWRSS], where the notation $a:=\frac{q}{m}$ is used. The zero level set of the momentum map is given by

$$
\mathbf{J}_{\mathfrak{s}^{*}}^{-1}(0)=\left\{\left(\mathbf{m}, \rho \frac{q}{m}, \rho, \sigma, A, Y\right) \mid \operatorname{div} \mathbf{E}=\rho \frac{q}{m}, \mathbf{E}:=-Y^{\sharp}\right\} .
$$

The bijection $i$ reads

$$
i\left(\mathbf{m}, \rho \frac{q}{m}, \rho, \sigma, A, Y\right)=\left(\mathbf{m}-A \rho \frac{q}{m}, \rho, \sigma, \rho \frac{q}{m}, A,-Y\right),
$$

and the image of $\mathbf{J}_{\mathfrak{s}^{*}}^{-1}(0)$ is given by

$$
\{(\mathbf{n}, \rho, \sigma, \nu, A, E) \mid \operatorname{div} \mathbf{E}=\nu\},
$$

where the notation $\mathbf{E}:=E^{\sharp} \in \mathfrak{X}(M)$ is used. The gauge transformation (4.12) is simply given by

$$
(\mathbf{n}, \rho, \sigma, \nu, A, E) \mapsto(\mathbf{n}, \rho, \sigma, \nu, A+\mathbf{d} \eta, E) .
$$

Through the diffeomorphism $i$, the projection $\pi_{0}: \mathbf{J}_{\mathfrak{S}^{*}}^{-1}(0) \rightarrow \mathbf{J}_{\mathfrak{s}^{*}}^{-1}(0) / \mathcal{G} a u(P)$ is given by

$$
(\mathbf{n}, \rho, \sigma, \nu, A, E) \mapsto(\mathbf{n}, \rho, \sigma,[A], E),
$$

where $[A] \in \Omega^{1}(M) / \mathbf{d} \mathcal{F}(M)$. Assuming that the first and second cohomology groups of $M$ are trivial, $H^{1}(M)=H^{2}(M)=\{0\}$, we get the isomorphism

$$
[A] \mapsto B:=\mathbf{d} A \in \Omega_{c l}^{2}(M),
$$

where $\Omega_{c l}^{2}(M)$ denotes the space closed two-forms. Thus $i$ induces a diffeomorphism between $\mathbf{J}_{\mathfrak{s}^{*}}^{-1}(0) / \mathcal{G} a u(P)$ and the space $\mathfrak{k}^{*} \times \Omega_{c l}^{2}(M) \times \Omega^{1}(M)$ given by

$$
[\mathbf{m}, \nu, \rho, \sigma, A, Y] \mapsto(\mathbf{m}-A \nu, \rho, \sigma, \mathbf{d} A,-Y)=:(\mathbf{n}, \rho, \sigma, B, E) .
$$

where $\Omega_{c l}^{2}(M)$ denotes the closed 2-forms on $M$. This identification coincides with the one given in Proposition 10.1 in [MWRSS].

Using the definition (4.15) and the bracket (4.14), the reduced Poisson bracket on $\mathfrak{k}^{*} \times \Omega_{c l}^{2}(M) \times \Omega^{1}(M)$ is given by

$$
\begin{aligned}
\{f, g\}_{0}(\mathbf{n}, \rho, \sigma, B, E)=\int_{M} \mathbf{n}\left(\left[\frac{\delta f}{\delta \mathbf{n}}, \frac{\delta g}{\delta \mathbf{n}}\right]_{L}\right) \mu \\
\quad+\int_{M} \rho\left(\mathbf{d}\left(\frac{\delta f}{\delta \rho}\right) \frac{\delta g}{\delta \mathbf{n}}-\mathbf{d}\left(\frac{\delta g}{\delta \rho}\right) \frac{\delta f}{\delta \mathbf{n}}\right) \mu \\
+\int_{M} \sigma\left(\operatorname{div}\left(\frac{\delta f}{\delta \sigma} \frac{\delta g}{\delta \mathbf{n}}\right)-\operatorname{div}\left(\frac{\delta g}{\delta \sigma} \frac{\delta f}{\delta \mathbf{n}}\right)\right) \mu \\
+\int_{M} g\left(\delta \frac{\delta g}{\delta B}, \frac{\delta f}{\delta E}\right) \mu-\int_{M} g\left(\delta \frac{\delta f}{\delta B}, \frac{\delta g}{\delta E}\right) \mu \\
+\int_{M} \rho \frac{q}{m}\left(\frac{\delta g}{\delta E}\left(\frac{\delta f}{\delta \mathbf{n}}\right)-\frac{\delta f}{\delta E}\left(\frac{\delta g}{\delta \mathbf{n}}\right)+B\left(\frac{\delta f}{\delta \mathbf{n}}, \frac{\delta g}{\delta \mathbf{n}}\right)\right) \mu
\end{aligned}
$$

and the Euler-Maxwell equations can be written as

$$
\dot{f}=\{f, h\}_{0},
$$

relative to the induced Hamiltonian $h$ given by

$h(\mathbf{n}, \rho, \sigma, B, E)=\frac{1}{2} \int_{M}\left(\frac{1}{\rho} g(\mathbf{n}, \mathbf{n})+\frac{1}{\rho}(\delta E)^{2}\right) \mu+\int_{M} \rho e(\rho, \sigma) \mu+\frac{1}{2} \int_{M}\left(\|E\|^{2}+\|B\|^{2}\right) \mu$. 
Note that the function

$$
C(\mathbf{n}, \rho, \sigma, B, E)=\frac{1}{2} \int_{M} \frac{1}{\rho}(\delta E)^{2}
$$

is a Casimir function, so an equivalent Hamiltonian is given by

$$
\bar{h}(\mathbf{n}, \rho, \sigma, B, E)=\frac{1}{2} \int_{M} \frac{1}{\rho} g(\mathbf{n}, \mathbf{n}) \mu+\int_{M} \rho e(\rho, \sigma) \mu+\frac{1}{2} \int_{M}\left(\|E\|^{2}+\|B\|^{2}\right) \mu .
$$

When $M$ is three dimensional, we can use the notations $\mathbf{B}:=(\star B)^{\sharp}$ and $\mathbf{E}:=$ $E^{\sharp}$. Therefore the two last terms can be written as

$$
\begin{aligned}
& \int_{M} g\left(\operatorname{curl} \frac{\delta g}{\delta \mathbf{B}}, \frac{\delta f}{\delta \mathbf{E}}\right) \mu-\int_{M} g\left(\operatorname{curl} \frac{\delta f}{\delta \mathbf{B}}, \frac{\delta g}{\delta \mathbf{E}}\right) \mu \\
& \quad+\int_{M} \rho \frac{q}{m}\left(g\left(\frac{\delta g}{\delta \mathbf{E}}, \frac{\delta f}{\delta \mathbf{n}}\right)-g\left(\frac{\delta f}{\delta \mathbf{E}}, \frac{\delta g}{\delta \mathbf{n}}\right)+g\left(\mathbf{B}, \frac{\delta f}{\delta \mathbf{n}} \times \frac{\delta g}{\delta \mathbf{n}}\right)\right) \mu .
\end{aligned}
$$

This bracket coincides with the one derived in [MWRSS] by a direct computation. Note that the first line in the formula above is the Pauli-Born-Infeld Poisson bracket for the Maxwell equations (see, e.g. [MR], §1.6). The Hamiltonian $\bar{h}$ is very simple: it is the sum of the total energy of the fluid plus the energy of the electromagnetic field.

\section{References}

[AM] R. Abraham and J. E. Marsden, Foundations of mechanics, Benjamin-Cummings Publ. Co, Updated 1985 version, reprinted by Perseus Publishing, second edition, 1978.

[A] V. I. Arnold, Sur la géométrie différentielle des groupes de Lie de dimenson infinie et ses applications à l'hydrodynamique des fluides parfaits, Ann. Inst. Fourier, Grenoble 16 (1966), 319-361.

[B] D. Bleecker, Gauge theory and variational principles, Addison-Wesley Publ. Co., 1981.

[EM] D. G. Ebin and J. E. Marsden, Groups of diffeomorphisms and the motion of an incompressible fluid, Ann. of Math. 92 (1970),102-163.

[GHK] Gibbons, J., D. D Holm and B. Kupershmidt, The Hamiltonian structure of classical chromohydrodynamics, Physica D 6 (1983), 179-194.

[HMR] Holm D. D, J. E. Marsden and T. S. Ratiu, The Euler-Poincaré equations and semidirect products with applications to continuum theories, Adv. in Math. 137 (1998), 1-81.

[KN] S. Kobayashi and K. Nomizu, Foundations of differential geometry, Wiley, 1963.

[MMOPR] J. E. Marsden, G. Misiołek, J.-P. Ortega, M. Perlmutter, and T. S. Ratiu Hamiltonian reduction by stages, Springer Lecture Notes in Mathematics, Springer-Verlag, 2007.

[MR] J. E. Marsden and T. S. Ratiu, Introduction to mechanics and symmetry, Texts in Applied Mathematics, 17, Springer-Verlag, 1994; Second Edition, 1999, second printing 2003.

[MRW] J. E. Marsden, T. S. Ratiu, and A. Weinstein, Semidirect product and reduction in mechanics, Trans. Amer. Math. Soc. 281 (1984), 147-177.

[MW] J. E. Marsden and A. Weinstein, The Hamiltonian structure of the Maxwell-Vlasov equations, Physica D 4 (1982), 394-406.

[MWRSS] J. E. Marsden, A. Weinstein, T. S. Ratiu, R. Schmid, and R. G. Spencer, Hamiltonian system with symmetry, coadjoint orbits and Plasma physics, Atti della Acad. della Sc. di Torino, Supplemento al Vol. 117, Proc. IUTAM-ISIMM Symposium on Modern Developments in Analytical Mechanics (Academy of Sciences Turin, Turin, June 7-11, 1982), 1983 pp. 289340 .

[M] R. Montgomery, Canonical formulations of a classical particle in a Yang-Mills field and Wong's equations, Lett. Math. Phys., 8 (1984), 59-67.

Section de Mathématiques and Bernoulli Center, École Polytechnique Fédérale De Lausanne, CH-1015 Lausanne, Switzerland

E-mail address: francois.gay-balmaz@epfl.ch, tudor.ratiu@epfl.ch 